Check for updates

Cite this: J. Mater. Chem. C, 2021, 9, 12951

DOI: $10.1039 /$ d1tc90195h

rsc.li/materials-c

\section{Correction: Highly efficient bilateral doping of single-walled carbon nanotubes}

\author{
Anastasia E. Goldt, ${ }^{a}$ Orysia T. Zaremba, ${ }^{a}$ Mikhail O. Bulavskiy, ${ }^{a}$ Fedor S. Fedorov, ${ }^{a}$ \\ Konstantin V. Larionov, ${ }^{\text {bc }}$ Alexey P. Tsapenko, ${ }^{d}$ Zakhar I. Popov, ${ }^{\text {be }}$ Pavel Sorokin, \\ Anton S. Anisimov, ${ }^{f}$ Heena Inani, ${ }^{9}$ Jani Kotakoski, ${ }^{9}$ Kimmo Mustonen ${ }^{g}$ and \\ Albert G. Nasibulin*ah
}

Correction for 'Highly efficient bilateral doping of single-walled carbon nanotubes' by Anastasia E. Goldt et al., J. Mater. Chem. C, 2021, 9, 4514-4521, DOI: 10.1039/D0TC05996J.

The authors regret the omission of a grant number (No. K2-2020-023) from the Acknowledgements section of the published article. The grant no. K2-2020-023 should have been be given for the theoretical work supported by the Ministry of Education and Science of the Russian Federation in the framework of Increase Competitiveness Program of NUST "MISiS".

The Royal Society of Chemistry apologises for these errors and any consequent inconvenience to authors and readers.

\footnotetext{
${ }^{a}$ Skolkovo Institute of Science and Technology, 3 Nobel Street, Moscow 121205, Russia. E-mail: a.goldt@skoltech.ru, a.nasibulin@skoltech.ru

${ }^{b}$ National University of Science and Technology "MISiS", Leninsky Prospect 4, Moscow 119049, Russian Federation

${ }^{c}$ Moscow Institute of Physics and Technology, Institutskiy Lane 9, Dolgoprudny, Moscow 141700, Russian Federation

${ }^{d}$ Aalto University, Department of Applied Physics, Puumiehenkuja 2, Espoo 00076, Finland

${ }^{e}$ Emanuel Institute of Biochemical Physics RAS, Moscow 119334, Russian Federation

${ }^{f}$ Canatu Ltd, Konalankuja 5, Helsinki 00390, Finland

${ }^{g}$ Faculty of Physics, University of Vienna, Boltzmanngasse 5, Vienna 1090, Austria

${ }^{h}$ Aalto University School of Chemical Engineering, Kemistintie 1, Espoo 16100, Finland
} 